https://doi.org/10.26480/asm.02.2017.16.19

\title{
EVALUATION OF COSMETICS FOR THEIR POTENTIAL CONTAMINANTS AND DRUG RESIST ANT MICROORGANISMS
}

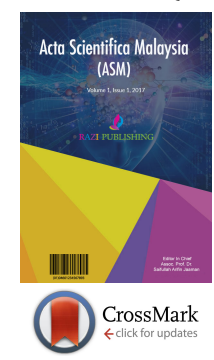

\author{
Saba Aslam*, Sajjad Ur Rahman, Zara Sabir, Bakhtawar Maqbool \\ Institute of Microbiology, Faculty of Veterinary Science, University of Agriculture, Faisalabad \\ *Corresponding Author Email: sabaaslam3964@gmail.com
}

This is an open access article distributed under the Creative Commons Attribution License, which permits unrestricted use, distribution, and reproduction in any medium, provided the original work is properly cited.

\section{ARTICLE DETAILS}

Article history:

Received 19 October 2017 Accepted 19 October 2017 Available online 25 October 2017

Keywords:

Microorganism, contaminants, cosmetics

\begin{abstract}
Cosmetic products are those formulations which are applied on different parts of body including teeth, nails, face and hair. These are used to clean body and improve appearance. On the basis of area on which cosmetics are applied these can be categorized in different categories like cosmetics for oral care, for skin, for hair and perfumes etc. Microorganisms are known for so many years due to their ability to grow and multiply in cosmetic care products. Cosmetic products are not considered to be sterile but there must be a minimum level of microbial contamination This study was conducted to assess the cosmetic care products for their potential microbial contaminants (both fungal and bacterial). Cosmetic products were collected in two categories; cosmetic creams and moisturizing lotions. Pour plate technique was used to determine total viable count. Sample dilution was made in Phosphate buffer saline containing $0.5 \%$ of an emulsifying agent that was polysorbate 80 . Different general purpose, selective and differential media were used for culturing. Identification was based upon morphology of colonies, Gram reaction and standard biochemical tests. Antibiotic sensitivity was checked by using different antibiotic and antifungal discs on MullerHinton Agar and Sabouraud Dextrose Agar. In this study the contaminants isolated from creams and lotions were; Pseudomonas aeruginosa, E. coli, Staphylococcus aureus and Aspergillus niger. Among these isolates P. aeruginosa, S aureus and E. coli were sensitive to Ciprofloxacin, Amikacin and Tobramycin while A. niger was sensitive to Streptomycin, Ketoconazole and Clotrimazole.
\end{abstract}

\section{INTRODUCTION}

In our society beauty standards are becoming very high due to the placement of photo shopped advertisements of models. These kinds of ads create a feeling of inadequacy among females. In a report named 'Beauty at any cost" which was created in 2008 by USA YWCA, The World Young Women's Christian Association, different consequences of women's obsessions with beauty were discussed. It was reported that these kinds of obsessions result in lack of self-esteem. Due to this reason, every year 7 billion dollars are spent on cosmetics in America [1].

In recent decades, the standards of human livings have been raised with a high demand for cosmetic products. In Malaysia almost 1255 million RM are spent for the import of these personal care cosmetic products, makeup products, hair products, fragrances as well as skin care products. Similarly, almost 592 million RM are spent on the export of these products [2]. In 2011 Brazil was known as the third largest country for using cosmetic items, perfumes and personal care products. The revenue recorded for Brazil was 43028.5 million US dollars. Out of this $7.3 \%$ was recorded for cosmetics [3].

Cosmetics are also called makeup products. These are known as the items which are sprayed, rubbed or sprinkled to any part of the body to clean and to increase the beauty. These items are used to enhance beauty and to change the look [4]. These are the substances which are meant to be used for the improvement of the appearance of human body. Cosmetics have gained great importance. Study of microbial contamination in these products is important because they have a direct contact with the human skin. Cosmetics are used regularly by a number of people and every year this number is getting increased [5]. Cosmetic products not only beautify the face but also release stress in females as well as hide the imperfections of face and enhance a women's personality. These products must be safe and easy in use. There are certain infections reported due to use of such products. These include allergies and different skin reactions along with inflammation [3].

Microorganisms are known for so many years due to their ability to grow and multiply in cosmetic care products [6]. Skin is outer most covering which provide protection. It acts as a barrier against pathogens [7]. Naturally present mechanical barriers and different mechanisms of defense protect human skin and mucosal membrane from microbial attack. This defense mechanism may get disturbed due to the application of cosmetic products. Although skin has a natural protective barrier but by using cosmetic products various ingredients can penetrate skin and becomes available systematically. Some of these products are applied on lips and they may get ingested orally. So, the safety of these products is very important. Contaminated cosmetics are hazardous to health. When the microorganisms exceed the limit specified by health care organizations, their use on topical areas may cause serious diseases and complications related to skin like; eczema, scabies, acne, dyschromia and many more [8]. The easiest way to evaluate risk factors related to the use of cosmetic products is to assess their exposure with the skin. Use of these beauty products is different according to age groups and geographical area. Their pattern of being used is also different [9].

Cosmetic products consist of many chemicals as well as a sufficient amount of water along with it. The countries where warm climatic conditions prevail, cosmetics are kept at room temperature. A majority of such products have almost neutral $\mathrm{pH}$. Although these products are preserved effectively even then there are chances of contamination. The results of such contamination may not be good for health [10]. Microbiologists have isolated different opportunistic microbes like Serratia spp., Klebsiella pneumoniae, Enterobacter spp., Pseudomonas aeruginosa and otherPseudomonas spp., since 1960 [11].

During the process of production, handling of raw material and by repeatedly using, these products can get contaminated. To withstand this problem a broad range of preservatives is developed [12]. Nowadays an art is involved to maintain equilibrium between the safety against contaminating microbes and health issues related to preservatives [13]. The main reason due to which cosmetic products are recalled is the microbiological contamination of these products [14]. Conditions which highly support the contamination caused by microorganisms are; usage frequency of cosmetic products, methods by which these are applied and conditions at which these are stored [15]. Formaldehydes are used as preservatives in cosmetic products. Their repeated use cause allergic reactions. Although in these products, formaldehydes are added in a very minute amount which does not cause any harmful effects unless used repeatedly. By using such products for long time, different sensitivity reactions may occur. It is used as a preservative in body soaps, nail paints, hair smoothing products, nail glues, baby shampoos and hair gels etc [16].

An important and necessary requirement for the production of cosmetics is having a starting material with low levels of microbial count usually lower than 10 Colony Forming Unit/g (CFU/g). The microbial counts which are mostly suggested for production areas are $500 \mathrm{cfu} / \mathrm{m} 3[17]$. The mission of an industrial microbiologist is elimination of contamination from cosmetic products up to possible level and to prevent the supply of such contaminated products in the market. So that he involves in all the stages of cosmetic production until the complete production. He also performs the final challenge test for cosmetics evaluation [18]. The infections which occur in some particular situations are of great importance for example; use of cosmetics around eye area, on skin when it is damaged, on children 
on children of age group 3 or less than 3 , on older people and on people with weak immune system. The pathogenic microorganisms which cause infection related to eyes due to application of cosmetic products are; Pseudomonas aeruginosa, Staphylococcus aureus, Klebsiella pneumoniae, Streptococcus pyogenes and other Pseudomonas spp. In cosmetic products, presence of microorganisms like; E. coli, Staphylococcus aureus, Clostridium spp., Salmonella spp., and Pseudomonas aeruginosa is strictly avoided [19].

\section{MATERIALS AND METHODS}

\subsection{Sample Collection}

Six samples consisting of two categories of cosmetic products representing 3 different $\mathrm{BB}$ creams and 3 different moisturizing lotions were employed in this study. All the samples were purchased from the local market of Faisalabad city. All the samples were unused. Their date of production, expiry date, batch number and added preservatives were carefully noted down. Creams were named as C1, C2 and C3. Similarly, lotions were named as L1, L2 and L3.

\subsection{Sample Processing}

After collection, each sample was dispersed into separate sterilized Phosphate buffer saline (PBS) containing $0.5 \%$ polysorbate 80 which is yellow colored viscous liquid. It is used as an emulsifying agent. For each sample, a 10-fold serial dilution was made under aseptic conditions. 10 sterilized test tubes were taken in a rack and labeled according to the dilution of its content. $9 \mathrm{ml}$ PBS containing polysorbate 80 was added in each test tube. $1 \mathrm{ml}$ from first sample was transferred to 1 st test tube and mixed thoroughly. After that $1 \mathrm{ml}$ solution from 1 st test tube was taken with a pipette and mixed in 2 nd test tube. Then $1 \mathrm{ml}$ from 2 nd test tube is transferred to 3 rd test tube and so on until the last dilution. $1 \mathrm{ml}$ from last test tube was discarded. Same procedure was done for all 6 samples [20].

\subsection{Media used}

Two general purpose media; Trypticase soy agar (TSA) for bacteria and Sabouraud dextrose agar (SDA) for fungi were prepared.

\subsection{Pour plate technique}

For the determination of total viable microbial count for each sample, pour plate method was used. First of all, sterilized Petri plates were taken. All the plates were labeled. $1 \mathrm{ml}$ solution from 2 nd test tube was taken and poured into the Petri plate. After that $15-20 \mathrm{ml}$ of sterile TSA was poured and mixed properly with that dilution. Same procedure was done for 106 and 108 dilutions. Fungi were isolated on SDA by using same method. Solidified TSA plates were incubated for $48 \mathrm{hrs}$ at $37^{\circ} \mathrm{C}$. SDA plates after solidification were incubated for almost 7 days at $27^{\circ} \mathrm{C}$. Each dilution was plated out in triplicates. After incubation, the numbers of colonies were counted by using colony counter. As all the dilutions were plated out in triplicates, so mean value for each dilution was taken after counting colonies separately.

\subsection{Viable microbial count}

For viable count pour plate method was used. After incubation colonies from TSA and SDA plates were counted by using colony counter. Mean of triplicates were taken to estimate total colonies grown from each dilution. Those samples were considered contaminated in which the colony count exceeded 300. To estimate the viable microbial count per milliliter of sample dilution, colony count was multiplied with appropriate dilution factor. All the values were obtained in $\mathrm{CFU} / \mathrm{ml}$ [21].

\subsection{Microbial identification}

All the bacterial isolates were identified on the basis of colony morphology, Gram staining and standard biochemical tests. Further examination of the organism was carried out by using different selective and differential media. For fungal isolates, Slide culture technique was used for identification.

\subsection{Pure Culture}

After colony counting further streaking of fungal colonies was done on SDA. Bacterial colonies were further streaked on different selective and differential media including MacConkey agar (MCA), Cetrimide agar (PCA), Staph 110 agar and Eosin methylene blue agar (EMB).

\subsection{Biochemical Test}

All the biochemical test was performed including; Gram staining, catalase test, coagulase test, oxidase test, Indole test, Methyl red, Vogesproskauer test and Citrate test for bacterial identification

\subsection{Antibiotic Susceptibility}

This was done to check the sensitivity and resistance of the bacterial and fungal isolates against different antibiotics and antifungal. This was determined by disc diffusion method. Media used for susceptibility testing of bacterial isolates was Muller Hinton Agar (MHA) and for fungal isolates SDA was used. MHA was prepared and poured in sterile petri plates. Bacterial and fungal cultures, which were inoculated in different test tubes containing broth, were kept overnight. Then sterile swabs were taken and dipped in this broth culture and the swabbing on culture plates was done. After that with the help of a sterile forceps antibiotic and antifungal discs were placed on these plates and incubation of $24 \mathrm{hrs}$ was provided. The results were interpreted in the form of diameter of zone of inhibition (ZDI). Zones were formed around each disc in case of sensitivity of that isolate against corresponding antibiotic. Result interpretation was done by following CLSI (Clinical Laboratory Standards Institute) criteria [22].

\section{RESULTS AND DISCUSSION}

In this study, the contaminants isolated from creams and lotions were $\mathrm{P}$. aeruginosa, S. aureus, E. coli and A. niger. P. aeruginosa, S. aureus and E. coli were sensitive to ciprofloxacin amikacin and tobramycin while A. niger was found sensitive to all three drugs used in this study.

Table 1: Microbial counts for bacteria (CFU/ml)

\begin{tabular}{|c|c|c|c|c|c|}
\hline Sr. no. & Samples & $\begin{array}{l}\text { Dilution } \\
\text { factor }\end{array}$ & $\begin{array}{l}\text { Av. Bacterial } \\
\text { count }\end{array}$ & $\mathrm{CFU} / \mathrm{ml}$ & Mean \\
\hline \multirow{3}{*}{1} & \multirow{3}{*}{$\mathrm{C} 1$} & $10^{2}$ & 314 & $3.1 \times 10^{4}$ & \multirow{3}{*}{$6.21 \times 10^{9}$} \\
\hline & & $10^{6}$ & 237 & $2.37 \times 10^{8}$ & \\
\hline & & $10^{8}$ & 184 & $1.84 \times 10^{10}$ & \\
\hline \multirow{3}{*}{2} & \multirow{3}{*}{ C2 } & $10^{2}$ & TNTC & ----- & \multirow{3}{*}{----- } \\
\hline & & $10^{6}$ & TNTC & ----- & \\
\hline & & $10^{8}$ & TNTC & ---- & \\
\hline \multirow{3}{*}{3} & \multirow{3}{*}{ C3 } & $10^{2}$ & 277 & $2.7 \times 10^{4}$ & \multirow{3}{*}{$3.56 \times 10^{9}$} \\
\hline & & $10^{6}$ & 191 & $1.91 \times 10^{8}$ & \\
\hline & & $10^{8}$ & 105 & $1.05 \times 10^{10}$ & \\
\hline \multirow{3}{*}{4} & \multirow{3}{*}{ L1 } & $10^{2}$ & TNTC & ----- & \multirow{3}{*}{----} \\
\hline & & $10^{6}$ & TNTC & ----- & \\
\hline & & $10^{8}$ & TNTC & ----- & \\
\hline \multirow{3}{*}{5} & \multirow{3}{*}{ L2 } & $10^{2}$ & 310 & $3.1 \times 10^{4}$ & \multirow{3}{*}{$5.83 \times 10^{9}$} \\
\hline & & $10^{6}$ & 213 & $2.13 \times 10^{8}$ & \\
\hline & & $10^{8}$ & 173 & $1.73 \times 10^{10}$ & \\
\hline \multirow{3}{*}{6} & \multirow{3}{*}{ L3 } & $10^{2}$ & 281 & $2.81 \times 10^{4}$ & \multirow{3}{*}{$3.35 \times 10^{9}$} \\
\hline & & $10^{6}$ & 160 & $1.60 \times 10^{8}$ & \\
\hline & & $10^{8}$ & 99 & $9.9 \times 10^{9}$ & \\
\hline
\end{tabular}

This table indicates the number of bacterial colonies counted with the help of colony counter. C1, C2 and C3 represent creams and L1, L2 and L3 represent lotions. In Lotion L1 and cream C2 colonies were too numerous to count. As all the dilutions were plated out in triplicates so mean value of each dilution $(102,106$ and 108) is mentioned in this table separately. To estimate the viable microbial count per milliliter of sample dilution, colony count was multiplied with appropriate dilution factor. Last column shows average of all these three dilutions. All the values were obtained in colony forming unit per $\mathrm{ml}(\mathrm{CFU} / \mathrm{ml})$.

Table 2: Microbial counts for fungi (CFU/ml)

\begin{tabular}{|c|c|c|c|c|c|}
\hline Sr. no. & Samples & $\begin{array}{l}\text { Dilution } \\
\text { factor }\end{array}$ & $\begin{array}{l}\text { Av. Bacterial } \\
\text { count }\end{array}$ & $\mathrm{CFU} / \mathrm{ml}$ & Mean \\
\hline \multirow{3}{*}{1} & \multirow{3}{*}{ C1 } & $10^{2}$ & 244 & $2.4 \times 10^{4}$ & \multirow{3}{*}{$3.92 \times 10^{9}$} \\
\hline & & $10^{6}$ & 176 & $1.7 \times 10^{8}$ & \\
\hline & & $10^{8}$ & 116 & $1.16 \times 10^{10}$ & \\
\hline \multirow{3}{*}{2} & \multirow[b]{2}{*}{ C2 } & $10^{2}$ & 209 & $2.09 \times 10^{4}$ & \multirow[b]{2}{*}{$2.13 \times 10^{9}$} \\
\hline & & \begin{tabular}{|l|}
$10^{6}$ \\
\end{tabular} & 106 & $1.06 \times 10^{8}$ & \\
\hline & & $10^{8}$ & 63 & $6.3 \times 10^{9}$ & \\
\hline \multirow{3}{*}{3} & \multirow{3}{*}{ C3 } & $10^{2}$ & No growth & No growth & \multirow{3}{*}{ No growth } \\
\hline & & $10^{6}$ & ----- & \begin{tabular}{|l|}
----- \\
\end{tabular} & \\
\hline & & $10^{8}$ & ------ & ----- & \\
\hline \multirow{3}{*}{4} & \multirow{3}{*}{ L1 } & $10^{2}$ & ----- & ----- & \multirow{3}{*}{----- } \\
\hline & & $10^{6}$ & ----- & \begin{tabular}{|l|}
$--{ }^{\prime}$ \\
--
\end{tabular} & \\
\hline & & $10^{8}$ & - ---- & \begin{tabular}{|l|}
----- \\
\end{tabular} & \\
\hline \multirow{3}{*}{5} & \multirow{3}{*}{ L2 } & $10^{2}$ & ------ & \begin{tabular}{|l|}
----- \\
\end{tabular} & \multirow{3}{*}{----- } \\
\hline & & $10^{6}$ & - & \begin{tabular}{|l}
--- \\
--
\end{tabular} & \\
\hline & & $10^{8}$ & ----- & \begin{tabular}{|l|}
---- \\
--
\end{tabular} & \\
\hline \multirow{3}{*}{6} & \multirow{3}{*}{ L3 } & $10^{2}$ & ------ & ------ & \multirow{3}{*}{----- } \\
\hline & & $10^{6}$ & ----- & \begin{tabular}{|l|}
----- \\
\end{tabular} & \\
\hline & & $10^{8}$ & ------ & |----- & \\
\hline
\end{tabular}


This table indicates the number of bacterial colonies counted with the help of colony counter. For cream C1 and C2, average values in terms of colony forming unit per $\mathrm{ml}$ are mentioned in last column. While, in cream C3, Lotion L1, L2 and L3 no fungal growth was observed. Identification of bacterial and fungal isolates was based on both macroscopic and microscopic morphology. Colonies present on TSA and SDA plates were first macroscopically observed. Mean values for all the dilutions were calculated. The colonies on TSA were creamy white and golden brown. On SDA plate colonies were creamy white in appearance. From TSA plates, further streaking was done on MacConkey agar, Staph 110 agar, Cetrimide agar and Eosin methylene blue agar and colony morphology was observed on all these plates. Similarly, from SDA plates further streaking was done on same media and morphology was observed. The growth of A. niger on SDA was creamy white to yellow in color initially when an incubation period of 2-3 days was provided but after 3-5 days it turned to carbon black colored spores. After Macroscopic examination, microscopic observation was also done for both bacterial and fungal isolates. Then Biochemical test including Indole test, MR test, VP test, Citrate test, Catalase test, Coagulase test, Oxidase test were also performed.

\subsection{Diagnosed Bacteria/Fungus}

All the samples showed contamination with varying degree. The isolate obtained from lotion L1 was E. coli. From L2 P. aeruginosa and from L3 S. aureus was isolated. S. aureus was found in all the creams. A. niger was isolated from C1 and C2. E. coli and P. aeruginosa was isolated from C3 and $\mathrm{C} 2$ respectively.

\subsection{Antibiotic and Antifungal Sensitivity}

To check antibiotic sensitivity against bacterial isolates (Pseudomonas aeruginosa, E. coli, and Staphylococcus aureus) antibiotics which were used included; Ciprofloxacin, Vancomycin, Erythromycin, Bacitracin, Tobramycin, Methycilin, Amikacin, Lincomycin and Colistinsulphate. For antifungal sensitivity Streptomycin, Ketoconazole and Clotrimazole were used.

Table 3: Results of Antibiotic sensitivity of bacterial isolates

\begin{tabular}{|l|l|l|l|}
\hline Drugs & P. a ruginosa & S. aureus & E. oli \\
\hline Ciprofloxacin & sensitive & sensitive & sensitive \\
\hline Vancomycin & resistant & resistant & resistant \\
\hline Erythromycin & resistant & sensitive & resistant \\
\hline Bacitracin & resistant & resistant & resistant \\
\hline Tobramycin & resistant & sensitive & sensitive \\
\hline Methycilin & resistant & resistant & resistant \\
\hline Amikacin & sensitive & sensitive & sensitive \\
\hline Lincomycin & resistant & resistant & resistant \\
\hline Colistinsulphate & sensitive & resistant & resistant \\
\hline
\end{tabular}

P. aeruginosa, S. aureus and E. coli were sensitive to ciprofloxacin amikacin and tobramycin while A. niger were sensitive to all three drugs used in this study.

Table 4: Results of Antifungal sensitivity

\begin{tabular}{|l|l|}
\hline Drugs & A. iger \\
\hline Streptomycin & Sensitive \\
\hline Ketoconazole & Sensitive \\
\hline Clotrimazole & Sensitive \\
\hline
\end{tabular}

\section{CONCLUSION}

This study was conducted to assess the cosmetic care products for potential microbiological contaminants (both fungal and bacterial). Antibiotic susceptibility of the isolates was also checked. Cosmetic products were collected in two categories; BB creams and moisturizing lotions. Pour plate technique was used to determine total viable count. Sample dilution was made in PBS containing $0.5 \%$ of an emulsifying agent that was polysorbate 80. Different general purpose, selective and differential media were used for culturing. Identification was based upon morphology of colonies, Gram reactions and standard biochemical test.
Antibiotic sensitivity was checked by using different antibiotics and antifungal discs on MHA and SDA. In this study the bacterial counts ranged from $6.21 \times 109$ to $3.35 \times 109 \mathrm{CFU} / \mathrm{ml}$ whereas, fungal counts ranged from $3.92 \times 109$ to $2.13 \times 109 \mathrm{CFU} / \mathrm{ml}$. Bacterial contamination was shown by all samples while two samples showed fungal contamination among total 6 samples. From creams and lotions, the bacteria isolated were; S. aureus, E. coli, and P. aeruginosa. While A. niger was only isolated from two creams $\mathrm{C} 1$ and $\mathrm{C} 2$. All these isolates showed high resistance against vancomycin, bacitracin, methycilin and lincomycin but ciprofloxacin and amikacin were highly active against all bacterial isolates. A. niger showed high sensitivity against all antifungals applied. This study indicated that all the samples were contaminated up to varying degree. Hence these products are potential cause of many skin diseases so that their use should be avoided.

\section{REFERENCES}

[1] Beauty at any cost. 2008. A YWCA report on the consequences of America's beauty obsession on women and girls.

[2] Afieza, K. 2000. Perkembangan Industri Kosmetik. Majalah Wanita, 9-11.

[3] Draelos, Z.D. 2001. Special considerations in eye cosmetics. Clinics in Dermatology, 19, 424-430.

[4] Nigam, P.K. 2009. Adverse reaction to cosmetics. Indian Journal of Dermatology, Venereology and Leprology, 75, 10-18.

[5] Hashim, P., Shahab, N., Masilamani, T. 2009. A Cosmetic Analysis in Compliance with the Legislative Requirements, Halal and Quality Control. Malaysian Journal of Chemistry, 11, 81-87.

[6] Fujital, P.G., and Onyerad, A. 2005. Microbial contamination and preservation capacity of some brands of cosmetic creams. Tropical Journal of Pharmaceutical Research, 2, 229-234.

[7] Templeton, K., Gripentrog, E., and Hellwig. T. 2012. Shining the Light on Sunscreen. U.S. Pharmacist, 37, 36-9.

[8] Mahé, A., Ly, F., Aymard, G. 2003. Skin diseases associated with the cosmetic use of bleaching products in women from Dakar, Senegal. British Journal of Dermatology, 148, 493-500.

[9] Ficheux, A.S., Wesolek, N., Chevillotteet, G. 2015. Consumption of cosmetic products by the French population. First part: frequency data. Food and Chemical Toxicology, 78, 159-169.

[10] Alvarez, L.F., Muall, E., Terradas, R. 2008. Moisturizing body milk as a reservoir of Burkholderiacepacia: outbreak of nosocomial infection in a multidisciplinary intensive care unit. Critical Care Medicine, 12, 10.

[11] Geis, P.A. 2006. Preservation strategies. Cosmetic microbiology, 2nd ed. Taylor \& Francis Group, New York, 163-180.

[12] NakiSiviri, N., Ozar, A.Y., Ozalp, M. 2006. Decontamination of cosmetic products and raw materials by gamma irradiation. FABAD Journal of Pharmaceutical Sciences, 198-209.

[13] Wu, Y.T., Zhu, H., Harmis, N.Y. 2010. Profile and frequency of microbial contamination of contact lens cases. Optometry and Vision Science Journal, $87,152-158$.

[14] Lundov, M., and Zachariae, C. 2008. Recalls of microbiologically contaminated cosmetics in EU from 2005 to May 2008. International Journal of Cosmetic Science, 30, 471-4.

[15] Gong, J.Q., Lin, L., Lin, T. 2006. Skin colonization by Staphylococcus aureus in patients with eczema and atopic dermatitis and relevant combined topical therapy: A double-blind multicentre randomized controlled trial. British Journal of Dermatology, 155, 680-7.

[16] Boyvat, A., Akyol, A., and G urgey, E. 2005. Contact sensitivity to preservatives in Turkey. Contact Dermatitis, 52, 333-337.

[17] Scholtyssek, R. 2004. Good manufacturing practice for producers of cosmetic ingredients. 10, 21-35.

[18] Russel, A.D. 2003. Challenge testing: principles and practice. International Journal of Cosmetic Science, 25, 147-153.

[19] Anthony, D.H., Tonyand, T.T., and James, E.M. 2001. Microbiological Methods for Cosmetics. Bacteriological Analytical Manual: Chapter 23. 
[20] Hugbo, P.G., Onyekweli, A.O., and Igwe, I. 2003. Microbial contamination and preservative capacity of some brands of cosmetic creams. Tropical Journal of Pharmaceutical Research, 2, 229-234.

[21] Peace, N.I.N., and Chinenye, C.C.O. 2016. Microbiological evaluation of cosmetics products sourced in Aba city, Nigeria. International Journal of Scientific Reports, 2, 74-80.

[22] Bauer, A.W., Kirbey, W.M., Sherris, J.C. 1966. Antibiotic susceptibility testing by a standard single diffusion method. American Journal of Clinical Pathology, 45, 493-496. 